\title{
Thermodynamic Consistency and Thermomechanical Dynamics (TMD) for Nonequilibrium Irreversible Mechanism of Heat Engines
}

\author{
Hiroshi Uechi ${ }^{*}$, Lisa Uechi ${ }^{2 *}$, Schun T. Uechi ${ }^{3 *}$ \\ ${ }^{1}$ Osaka Gakuin University, Suita, Japan \\ ${ }^{2}$ University of California, Los Angeles, USA \\ ${ }^{3}$ KPMG Ignition Tokyo, Data Tech., Tokyo, Japan \\ Email: ^uechi@ogu.ac.jp, „luechi@mednet.ucla.edu, ^schun.uechi@gmail.com
}

How to cite this paper: Uechi, H., Uechi, L. and Uechi, S.T. (2021) Thermodynamic Consistency and Thermomechanical Dynamics (TMD) for Nonequilibrium Irreversible Mechanism of Heat Engines. Journal of Applied Mathematics and Physics, 9, 1364-1390.

https://doi.org/10.4236/jamp.2021.96093

Received: May 19, 2021

Accepted: June 27, 2021

Published: June 30, 2021

Copyright $\odot 2021$ by author(s) and Scientific Research Publishing Inc. This work is licensed under the Creative Commons Attribution International License (CC BY 4.0).

http://creativecommons.org/licenses/by/4.0/

\begin{abstract}
The irreversible mechanism of heat engines is studied in terms of thermodynamic consistency and thermomechanical dynamics (TMD) which is proposed for a method to study nonequilibrium irreversible thermodynamic systems. As an example, a water drinking bird (DB) known as one of the heat engines is specifically examined. The DB system suffices a rigorous experimental device for the theory of nonequilibrium irreversible thermodynamics. The DB nonlinear equation of motion proves explicitly that nonlinear differential equations with time-dependent coefficients must be classified as independent equations different from those of constant coefficients. The solutions of nonlinear differential equations with time-dependent coefficients can express emergent phenomena: nonequilibrium irreversible states. The couplings among mechanics, thermodynamics and time-evolution to nonequilibrium irreversible state are defined when the internal energy, thermodynamic work, temperature and entropy are integrated as a spontaneous thermodynamic process in the DB system. The physical meanings of the time-dependent entropy, $T(t) \mathrm{d} \mathcal{S}(t)$, internal energy, $\mathrm{d} \mathcal{E}(t)$, and thermodynamic work, $\mathrm{d} W(t)$, are defined by the progress of time-dependent Gibbs relation to thermodynamic equilibrium. The thermomechanical dynamics (TMD) approach constitutes a method for the nonequilibrium irreversible thermodynamics and transport processes.
\end{abstract}

\section{Keywords}

Thermodynamic Consistency, Thermomechanical Dynamics (TMD), 
Nonlinear Differential Equation with Time-Dependent Coefficients, Nonequilibrium Irreversible States

\section{Introduction}

A water drinking bird (DB) is a delightful, scientific toy that produces a simple back and forth motion by occasionally dipping its beak in water as if it keeps moving only with water supply. On the contrary to the delicate and slender figure, the DB system is an excellent educational tool for mechanical and thermodynamic transport systems of heat and energy as Albert Einstein and many other scientists have been fascinated [1] [2] [3]. It demonstrates thermodynamic phenomena, or more precisely, irreversible thermodynamic phenomena. We have discussed the equation of motion and solution to the thermomechanical DB model and practical applications through thermoelectric energy conversions [4] [5]. Based on the papers, the irreversible thermodynamic DB system and thermodynamic consistency, time-evolution to thermodynamic equilibrium, the concept of coupling and decoupling of mechanical and thermodynamic systems will be discussed and numerically evaluated in the current paper.

The mathematical definition of thermodynamic equilibrium is given by the fundamental relation in thermodynamics [6] [7]:

$$
\left.\frac{\partial \mathcal{S}(\mathcal{E}, V)}{\partial \mathcal{E}}\right|_{V, N, \cdots}=\frac{1}{T},
$$

with the entropy $\mathcal{S}$, internal energy $\mathcal{E}$, temperature $T$, at fixed volume $V$ and particle number $N, \cdots$, at thermodynamic equilibrium (Boltzmann constant, $\left.k_{B} \equiv 1\right)$. The first and the second laws of thermodynamics and relations among thermodynamic functions are incorporated in Equation (1.1) at thermal equilibrium. The drinking bird begins with a simple pendulum motion of classical mechanics; gas in head-bulb and volatile water in a glass tube are at thermal equilibrium but gradually evolve to nonequilibrium irreversible states and then, the mechanism leads to a drinking motion. One may think that motion of the drinking bird is easy and simple, but one should be very careful that the drinking bird's motion develops from mechanical and thermodynamic equilibriums to a nonequilibrium irreversible state, which is one of the fundamental topics for the research and the laws of physics.

We employ the fundamental relation (1.1) for the time-evolution from mechanical and thermodynamic equilibriums to a nonequilibrium irreversible state and vice versa. The change of the thermal state is related to time-evolution of the internal energy, $\mathcal{E}(t)$, entropy $\mathcal{S}(t)$, temperature $T(t)$ and work $W(t)$ in the DB system, and the work $W(t)$ includes a time-change of volume $V(t)$. Hence, we suppose the transformation from mechanical and thermodynamic states to a nonequilibrium irreversible state (NIS) by: 


$$
\left.\frac{T \partial \mathcal{S}(\mathcal{E}, V)}{\partial \mathcal{E}}\right|_{V, N, \cdots}=\left.\left.1\right|_{t \leq t_{c}} \underset{t_{c}<t}{\longrightarrow} \frac{T(t) \partial \mathcal{S}(t, \mathcal{E}(t), W(t))}{\partial \mathcal{E}(t, W(t))}\right|_{t \leq t_{d}},
$$

where the time $t_{c}$ is a "critical time" (onset time) for the transition, and $t_{d}$ is the corresponding drinking (dipping) time. The transition from a NIS to an equilibrium state after dipping is similarly investigated by:

$$
\left.\left.\frac{T(t) \partial \mathcal{S}(t, \mathcal{E}(t), W(t))}{\partial \mathcal{E}(t, W(t))}\right|_{t \leq t_{d}} \underset{t_{d}<t}{\longrightarrow} \frac{T \partial \mathcal{S}(E, V)}{\partial \mathcal{E}}\right|_{V, N, \cdots}=1 .
$$

Though detailed heat conduction mechanisms should be introduced for transitions at $t_{c}$ and $t_{d}$ shown by arrows in (1.2) and (1.3), the transitions are mathematically simplified and expressed by using piecewise continuous function (the step function) in the thermomechanical model [4]. The "thermodynamic consistency of irreversible processes" is examined by conditions: (1.1)-(1.3).

The transition from equilibrium to a NIS starts at $t_{c}$ shown by (1.2), and a different mode of oscillation driven by thermodynamic work (up-going volatile water in the glass tube) is observed until a DB's dipping. After a DB's dipping, the volatile water in the glass tube is artificially returned to the lower bulb by a mechanical trick. The mathematical technicality corresponding to the mechanical trick is produced with the step function and termed as "initialization" [4]. The transition from a NIS to a thermodynamic equilibrium (1.3) is also shown consistently after DB's drinking time, $t_{d}$, which will be explained in detail in Section 2. One would realize that the DB system and motion can be a perfect experimental device to study fundamental laws of thermodynamics and nonequilibrium irreversible thermodynamics [8] [9] [10].

The time period, $t_{c}<t<t_{d}$, characterizes a NIS, and one can check different modes of oscillations (slow and elongated, slightly-increasing oscillations) developing to water drinking motion, and after drinking, the DB motion is decoupled to an equilibrium, which is composed of independent mechanical and thermodynamic states. The time-evolution from equilibrium to a NIS and then, to an equilibrium state can be clearly observed from the change of oscillation modes. In other words, the emergent coupling and decoupling of mechanical and thermodynamic states can be numerically measured in DB's oscillations. Although the nonlinear differential equation with constant coefficients is used as the DB's starting equation of motion by way of Hamiltonian (or Lagrangian) method, the DB motion cannot be reproduced by the nonlinear differential equation with constant coefficients. The mathematical equation of motion for the water drinking motion is a nonlinear differential equation with time-dependent coefficients, which has independent solutions to those of nonlinear differential equation with constant coefficients. The nonlinear differential equations with constant or time-dependent coefficients must be categorized as different classes of nonlinear differential equations, which is a mathematically important property discovered in the DB analysis, which will be explained in Section 2 and Section 3. 
The macroscopic (total or global) entropy, $\mathrm{d} \mathcal{S}$ and microscopic (constituent or local) entropies $\mathrm{d} \mathcal{S}_{i}$ have been often discussed in thermodynamic equilibrium, or linear and near-equilibrium states so that the entropy relation, $\mathrm{d} \mathcal{S}=\sum_{i} \mathrm{~d} \mathcal{S}_{i}$, holds. One should realize that fundamental complications will arise when the internal energy, work, heat, particle flows of photon, electron, nuclear particles, ..., are self-consistently coupled to each other in nonequilibrium and irreversible processes, resulting in $\mathrm{d} \mathcal{S} \neq \sum_{i} \mathrm{~d} \mathcal{S}_{i}$. This would be a reason why one needs to restrict thermodynamic variables for adequate descriptions of nonequilibrium systems [11]. One must determine the minimum number of thermodynamic variables that contributes decisively to the total system because not only constituents entropies but also internal energies and thermodynamic work would selfconsistently interact with all other constituents. The self-consistent interactions of one total entropy multiplied by temperature, $T(t) \mathcal{S}(t)$, internal energy $\mathcal{E}(t)$, work $W(t)$ are sufficient to study the DB system.

The terms, entropy, entropy production or dissipation, are known to be defined and used by different researchers in different ways, such as the maximum or minimum entropy production, minimum energy dissipation, etc., which is creating much confusion and logically circular arguments and statements [12] [13] [14] [15]. If the state of confusion is generated for not only beginners but also researchers, it should be improved for applications to accurate and delicate modern technologies of diverse fields, such as biological, medical, chemical and quantum transport systems [16] [17] [18] [19] [20]. The water drinking bird would be helpful as an enlightening and rigorous experimental device for studying a NIS.

Modern technologies are progressing towards higher speed, power, miniaturization, efficient energy use and minimization of waste of energy, necessary for supporting ecological diversity and energy sustainable societies. In addition, space technologies will demand high energy use and energy efficiency. The issues in diverse fields of different energy conversions, harvesting and storage technologies are important for modern society to solve existing environmental problems [21] [22]. The world's primary energy consumption is increasing and reported as 14 TW-years/year, and energy efficiencies of mechanoelectric or thermoelectric energy conversions have been actively investigated [23] [24] [25] for solar, biomass, geothermal, hydrodynamic and wind turbines, coal-fired and nuclear power plants. These energy generation technologies will require new and improved knowledge. The optimal and sensitive thermoelectric energy conversion method based on the DB mechanism [5] could be useful for technological analyses and applications.

The analysis of drinking bird made us study the fundamental structure for theories of science, which would also help people in science reflect on physical principles of sciences; it is summarized that the theory of science should be examined in terms of reproducibility, self-consistency and testability. These criteria are difficult to achieve in general for scientific theories and models, but it would be reasonable to say that the four fundamental mechanics (Analytical 
mechanics, Thermodynamics, Electromagnetism, Quantum mechanics) and associated models, such as special relativity, quantum electrodynamics (QED) seem to maintain, and they have shown reproducibility, theoretical self-consistency and testability, resulting in great contributions to modern technologies. The DB papers [4] [5] elucidates reproducibility and testability, which includes experimental and mathematical reproductions of observed data and possible applications in the field of thermoelectric energy conversions. The purpose of the paper is to study thermodynamic consistency of a drinking bird mechanism and a methodology for nonequilibrium irreversible thermodynamics.

The time-dependent internal energy $\mathcal{E}(t)$, nonlinear differential equation of motion with time-dependent coefficients, coupled and decoupled oscillations are discussed with a short review of the thermomechanical DB model in Section 2. Then, the time-evolution of internal energy $\mathcal{E}(t)$, work $W(t)$, total entropy $\mathcal{S}(t)$ and temperature $T(t)$, thermodynamic consistency of the DB system and heat engines are examined in Section 3. The foundation of thermomechanical dynamics (TMD) for nonequilibrium irreversible thermodynamics is discussed in Section 4. The conclusions and perspectives of scientific ways of thinking are discussed in Section 5.

\section{The Time-Dependent Internal Energy $\mathcal{E}(t)$ and the}

\section{Equation of Motion in the Irreversible Thermomechanical DB Model}

A drinking bird and its topological deformation and the angle $\theta(t)$ are shown in Figure 1(a) and Figure 1(b) respectively. The DB's thermodynamic equilibrium is defined by (1.1): $\operatorname{T} \partial \mathcal{S}(E, V) /\left.\partial \mathcal{E}\right|_{V, N, \ldots}=1$, and subsequently, the mechanical equilibrium must be defined before discussing the time-evolution of the internal energy, $\mathcal{E}(t)$, entropy $\mathcal{S}(t)$ and work $W(t)$. The starting equation of motion for a mechanical drinking bird should be constructed by employing Lagrangian or Hamiltonian [26] [27], and the total energy with the rotational kinetic energy and the potential energy defined from the axis of rotation is:

$$
\mathcal{E}=\frac{1}{2} I_{0} \dot{\theta}^{2}-\left\{m_{2} g a-m_{1} g\left(b-\frac{l}{2}\right)-m_{3} g b\right\} \cos \theta,
$$

where the DB's length is $l=a+b$. The moment of inertia is given by the sum, $I_{0}=I_{1}+I_{2}+I_{3}$, the moment of inertia of head $I_{3}$, glass tube $I_{1}$, and lower bulb $I_{2}$, respectively [4]. The moment of inertia of head and lower bulb are given by $I_{2}=m_{2} a^{2}$ and $I_{3}=m_{3} b^{2}$, and $I_{1}$ is given by,

$$
I_{1}=m_{1} l^{2}\left(\frac{1}{3}-\left(1-\frac{b}{l}\right)+\left(1-\frac{b}{l}\right)^{2}\right) .
$$

The effective mass $m^{*}$ is introduced for convenience as,

$$
m^{*}=m_{2} \frac{a}{l}-m_{1}\left(\frac{b}{l}-\frac{1}{2}\right)-m_{3} \frac{b}{l},
$$




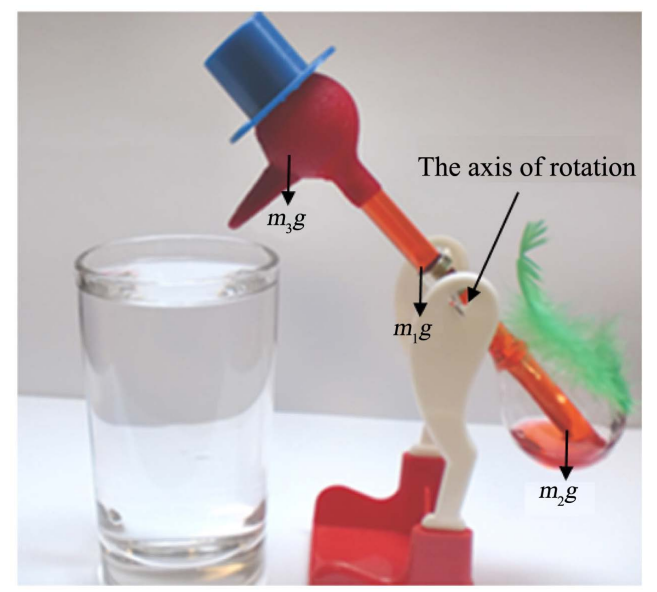

(a)

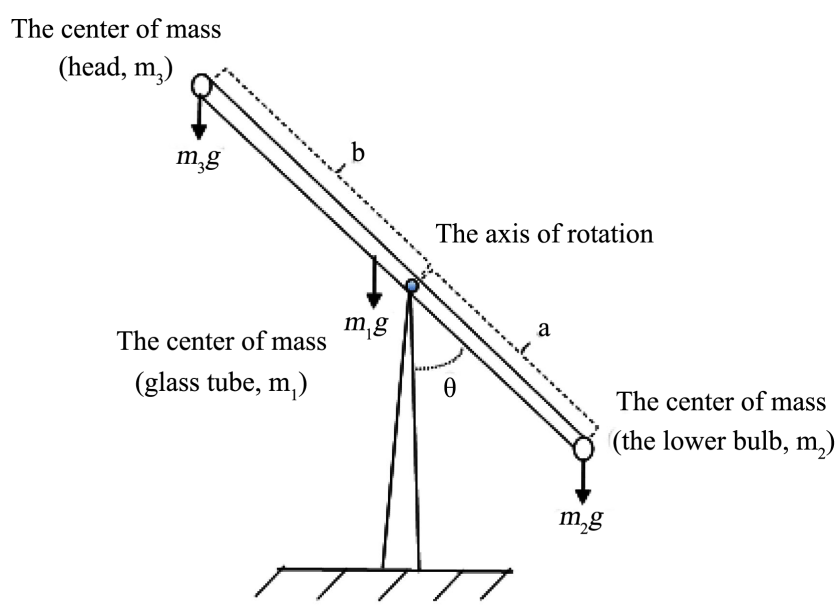

(b)

Figure 1. A mechanical modeling of a drinking bird. (a) A drinking bird (DB) devided into the head $m_{3}$, glass tube $m_{1}$, and bottom $m_{2}$ which includes volatile water; (b) A topological deformation for the drinking bird of (a) by employing the concept of centers of mass.

and the DB energy is written with $m^{*}$ as,

$$
E=\frac{1}{2} I_{0} \dot{\theta}^{2}-\operatorname{lgm}^{*} \cos \theta
$$

where the potential energy is measured from the axis of rotation in Figure 1(a), and so, the total energy can be negative or positive according to $\theta$ and $\dot{\theta}$. The mechanical equation of DB motion is written as,

$$
\ddot{\theta}+c \dot{\theta}+\frac{g l m^{*}}{I_{0}} \sin \theta=0 .
$$

The DB motion derived from (2.4) and (2.5) can express, for example, backand-forth solutions around $\theta=0$, or up-side-down solutions around $\theta=\pi$, as shown respectively in Figure 2(a). Generally speaking, the nonlinear Equation (2.5) has stable independent solutions converging to $\theta_{n}= \pm n \pi(n=0,1,2,3, \cdots)$, and the solution suddenly changes from the $\theta=0$ 


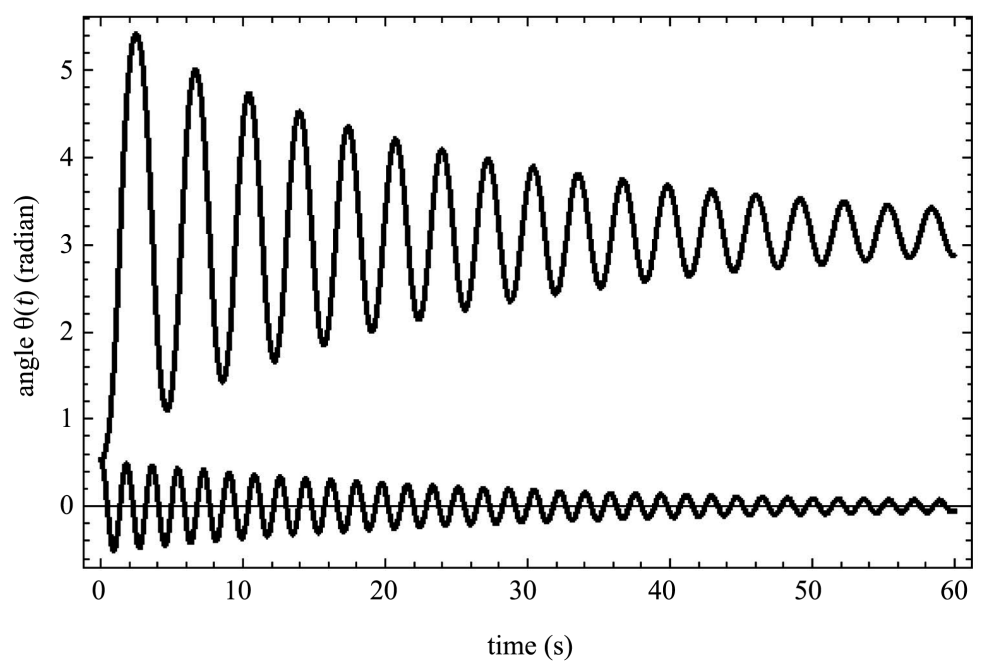

(a)

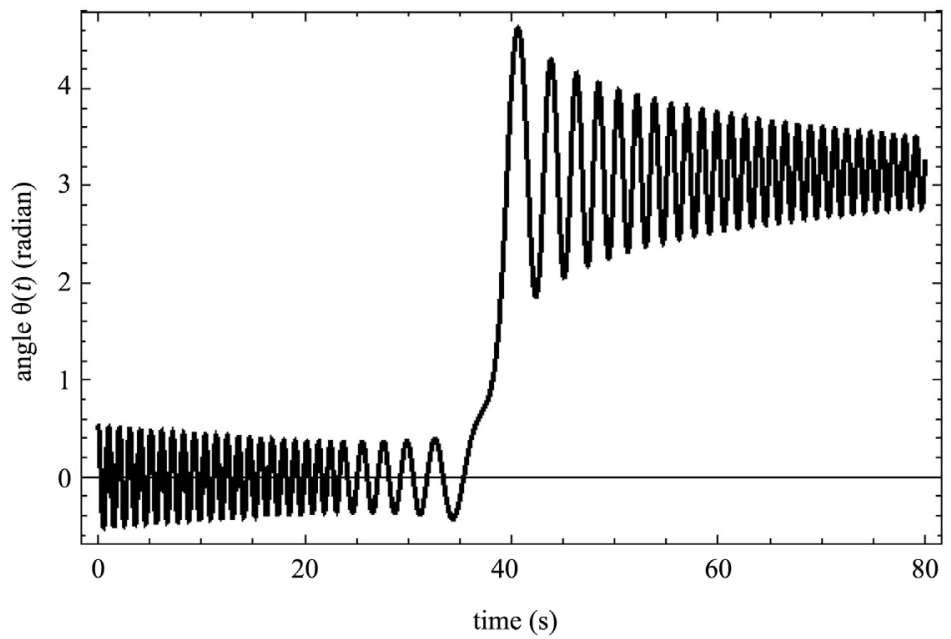

(b)

Figure 2. The solutions of nonlinear differential equations with constant and time-dependent coefficients. (a) The independent solutions of the nonlinear equation with constant coefficients (2.5), starting from $\theta=\pi / 6$, and converging to $\theta=0$ and $\theta=\pi$, respectively; (b) The solution of the nonlinear equation with time-dependent coefficients (2.7), which is the continuous solution starting from $\theta=\pi / 6$ and converging to $\theta=\pi$. The arrow points the drinking angle and time, $t_{d}$. The initialization (2.8) at $t_{d}$ produces Figure 3(a).

converging solution to the others when certain parameter values of mass $m$ and moment of inertia $I_{0}$ are given (other parameters, $g, l, c$, are fixed). The property of splitting into independent solutions is known as bifurcation phenomena for nonlinear differential equations with constant coefficients [28] [29], which proves that there are no continuous solutions changing from $\theta=0$ to $\theta=\pi$ in (2.5). The existence and derivations of exact values of parameters for bifurcation phenomena are not known. When the bird moves from $\theta=0$ to $\theta=\pi$ solution, the bird can drink water. Therefore, it is not possible to obtain a water drinking solution from the energy and equation of motion, (2.4) and (2.5). 
The up-going liquid in a glass tube changes the DB moment of inertia and internal energy, and we assumed a constant velocity, $v(t)=v_{0}$, for the up-going liquid [4]. The constant velocity, $v_{0}$, can be considered as an "averaged" velocity of up-going slightly-oscillating liquid, and time variations of $I(t)$ and $m^{*}(t)$ are determined. The numerical simulation is stable around a constant velocity, $v_{0}$, which is one of the reasons why the model calculation is successful. The time-dependent internal energy $\mathcal{E}(t)$ is given by,

$$
\mathcal{E}(t)=\frac{1}{2} I(t) \dot{\theta}^{2}-\operatorname{lgm}^{*}(t) \cos \theta,
$$

and detailed expressions of $I(t)$ and $m^{*}(t)$ are discussed in the paper [4]. The correct time-evolution equation of DB motion is,

$$
\ddot{\theta}+c \dot{\theta}+\frac{g l m^{*}(t)}{I(t)} \sin \theta=0
$$

The nonlinear differential Equations (2.6) and (2.7) should be compared with the energy and mechanical equation of motion (2.4) and (2.5), and numerical solutions are respectively shown in Figure 2(a) and Figure 2(b). The nonlinear differential Equation (NDE) with constant coefficients cannot produce the continuous solution in Figure 2(b). The NDE with time-dependent coefficients in (2.7) can reasonably produce the $\mathrm{DB}$ oscillations, and it is possible to generate a continuous solution converging from $\theta=0$ to $\theta=\pi$. Therefore, the nonlinear differential equation with constant coefficients and time-dependent coefficients must be mathematically categorized in a different class of nonlinear equations.

One should also note that the equation of motion (2.7) cannot be derived from (2.6) by way of a mechanical, Lagrangian or Hamiltonian method, because the energy (2.6) is explicitly time-dependent and equivalently non-conservative. However, the physical results and mechanism of NDE with time-dependent coefficients, the DB's time-dependent $m^{*}(t)$ and $I(t)$, driven by time-dependent energy $\mathcal{E}(t)$ and thermodynamic work $W(t)$ are consistent with DB's motion as a NIS. The arrow in Figure 2(b) points DB's water dipping angle, $\theta \sim \pi / 2$, and time, $t_{d} \sim 38.8(\mathrm{~s})$. The $\mathrm{DB}$ motion returns to a $(\theta=0)$-converging oscillation at $t_{d} \sim 38.8(\mathrm{~s})$, which is a mechanical trick with the artificial shape of arm and axis of rotation (see, Figure 1(a)). The mechanical trick to return to a ( $\theta=0$ )-converging oscillation is defined as "Initialization", and mathematically defined with a piecewise continuous step-function [4] as,

$$
\left(x_{2}(t), m^{*}(t), I(t)\right) \rightarrow\left(a, m^{*}, I_{0}\right)_{t_{d}},
$$

at a drinking time $t_{d}$. This is a mathematical simplification, which must be extended by including mechanism of heat conduction, diffusion and ignition phenomina [30] [31] [32] [33] [34].

The motion from the initial oscillations to the first critical time $t_{c} \sim 24.0$ (s), then to the dipping at $t_{d} \sim 38.8(\mathrm{~s})$ and oscillations after $t_{d}$ is shown in Figure 3(a). The DB oscillations are in thermal and mechanical equilibriums up to 


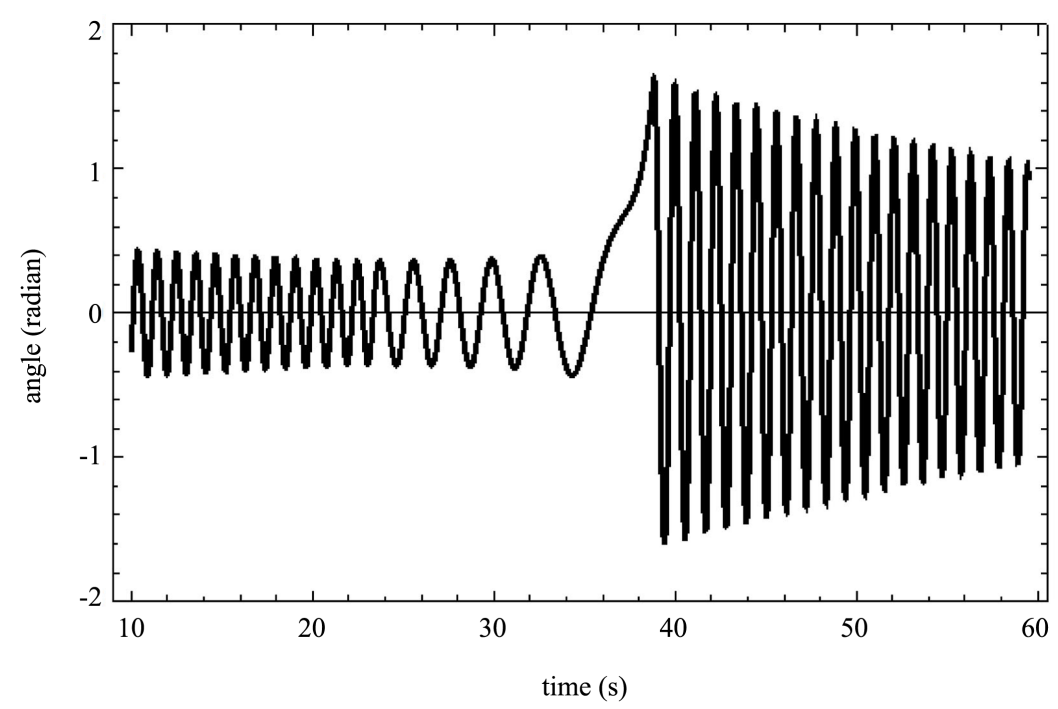

(a)

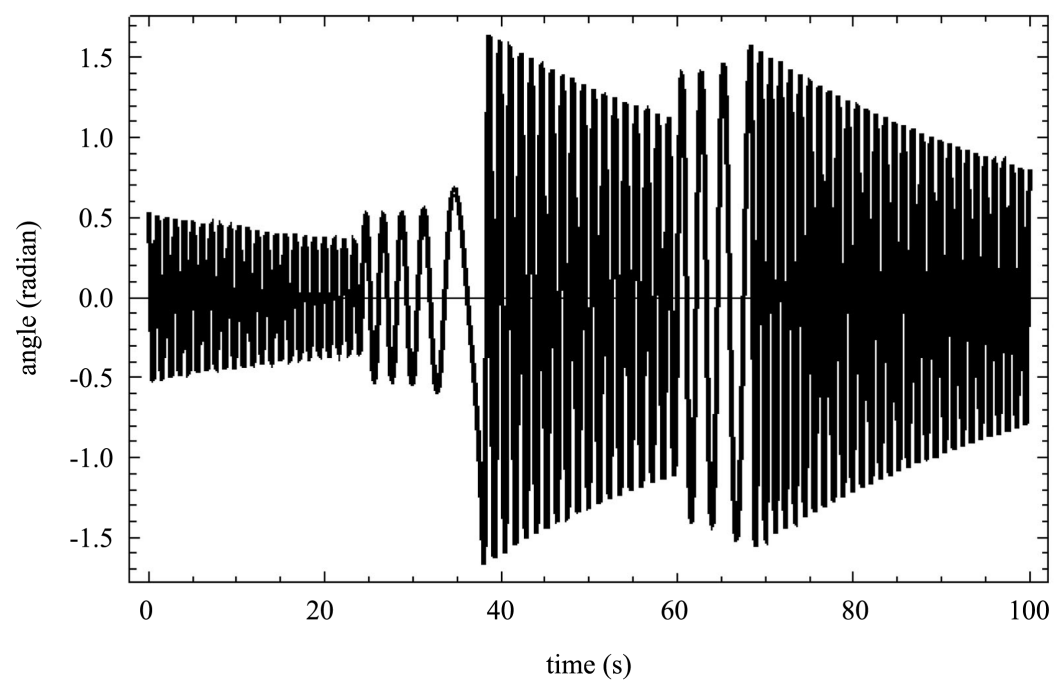

(b)

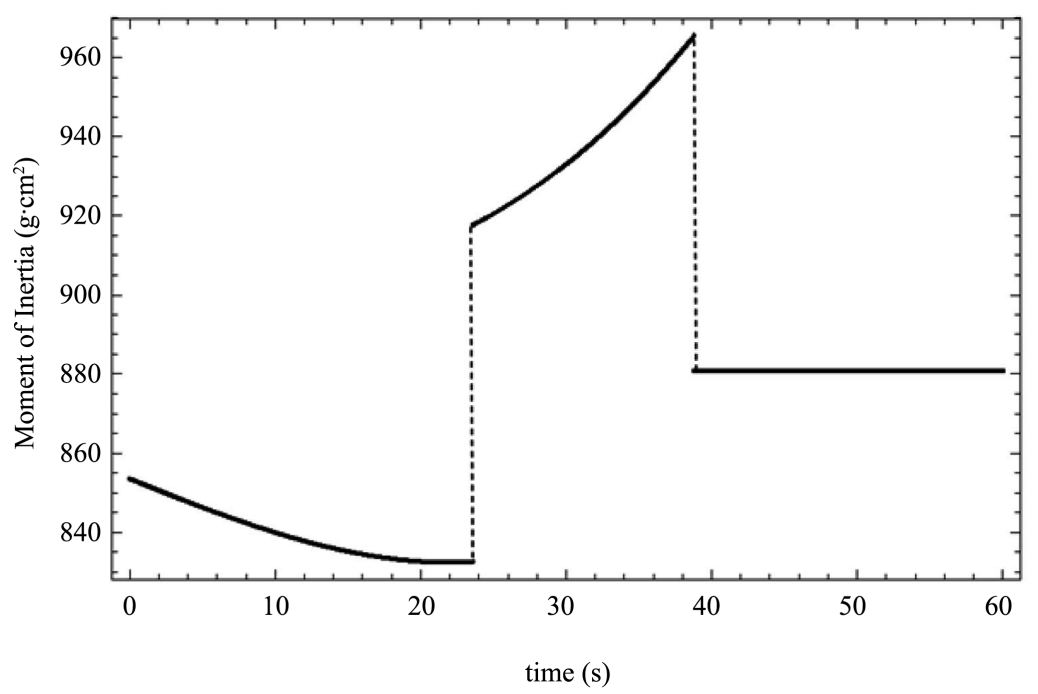

(c) 


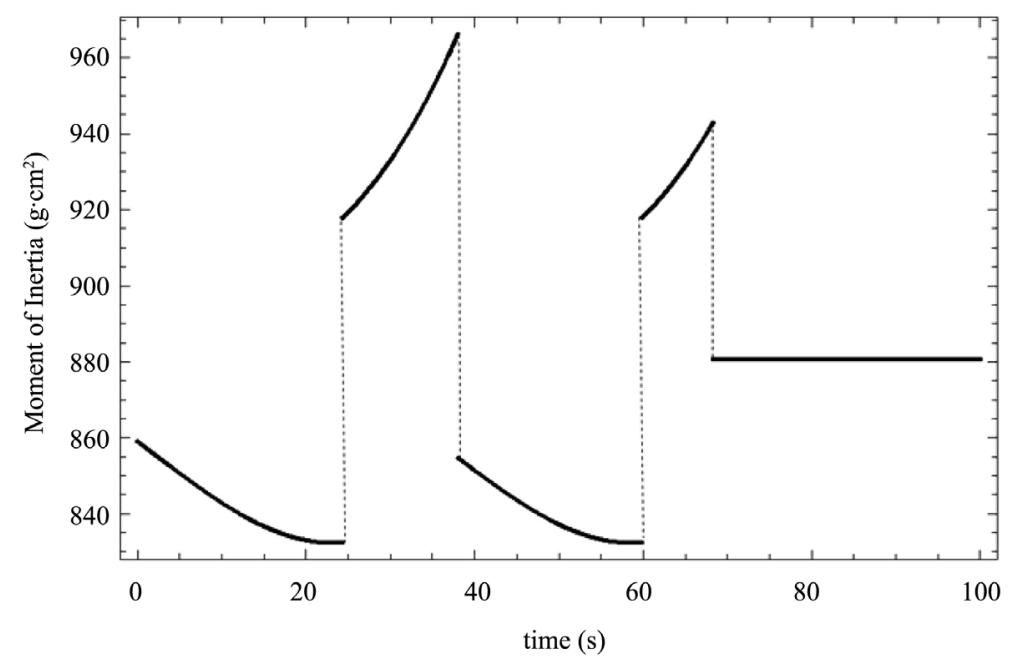

(d)

Figure 3. The drinking oscillations and the time-variation of moment of inertia, $I(t)$, in (2.6) and (2.7) (for detailed calculations, see the paper [4]). (a) The 1st forced-oscillation extending to water-drinking arises at $t_{1 c} \simeq 24.0(\mathrm{~s})$, and the bird's 1 st-drinking motion and initialization at $t_{1 d} \simeq 38.8(\mathrm{~s})$ and oscillations after drinking are shown; (b) The 1st forced-oscillation arises at $t_{1 c} \simeq 24.0(\mathrm{~s})$ and the bird's 1st-drinking motion at $t_{1 d} \simeq 38.0(\mathrm{~s})$. The second forced-oscillation at $t_{2 c} \simeq 60.5(\mathrm{~s})$, the 2nd-drinking at $t_{2 d} \simeq 68.3(\mathrm{~s})$ and oscillations after the drinking; (c) The time-variation of the moment of inertia $I(t)$ corresponding to (a). Note the corresponding change of $I(t)$ at $t_{1 c} \simeq 24.0(\mathrm{~s}) ;$ the 1 st-drinking and initialization $\left(I(t) \rightarrow I_{0}\right)$ at $t_{1 d} \simeq 38.8(\mathrm{~s}) ;$ (d) The time-variation of the moment of inertia $I(t)$ corresponding to $(b)$. The 1st forced-oscillation starting at $t_{1 c} \simeq 24.0(\mathrm{~s})$, drinking and initialization $\left(I(t) \rightarrow I_{0}\right)$ at $t_{1 d} \simeq 38.0(\mathrm{~s})$. The 2nd forced-oscillation starting at $t_{2 c} \simeq 60.5(\mathrm{~s})$, drinking and initialization at $t_{2 d} \simeq 68.3(\mathrm{~s})$.

a critical time $t_{1 c} \simeq 24.0(\mathrm{~s})$, and the change of oscillation-mode induced by thermodynamically-driven forced oscillations is observed from $t_{1 c}$ to $t_{1 d} \sim 38.8(\mathrm{~s})$. The critical time $t_{c}$ signifies the onset and transition from equilibrium to a NIS and the reversed transition occurs at the dipping time $t_{d}$. Similarly, the DB's 1st and 2nd oscillations are shown in Figure 3(b). The simple oscillations after $t_{1 d}$ reaches the 2nd critical time $t_{2 c} \sim 60.5(\mathrm{~s})$, and the 2nd slow and elongated oscillations develop up to the second dipping, $t_{2 d} \simeq 68.3(\mathrm{~s})$. After dipping at $t_{2 d}$, the DB system returns to mechanical and thermodynamic equilibriums. In short, the mechanical and thermodynamic states couple at the critical time, $t_{c}$ and emerge as a NIS, and then decouple to respective equilibrium states at $t_{d}$. The time periods of oscillations, $t_{1 c}<t<t_{1 d}$ and $t_{2 c}<t<t_{2 d}$, exhibit NISs.

The mechanical energy conservation law loses its meaning, but instead, the total conservation of heat and energy is restored in the NIS. The physical coefficients of the internal energy and equation of motion become time-dependent and progress to the NIS, which is fundamentally different from mechanical and 
thermal equilibriums. These phenomena are not supposed to be obtained from the continuous unitary-transformation in time in classical and quantum mechanical systems because time-symmetry is broken in a NIS. The time-dependence of thermodynamic work $W(t)$ is considered to be spontaneously produced and never infinitesimally slowly constructed in a NIS, and thermodynamic work also depends on levels of technology to convert the thermal energy. The physical mechanism to induce time-dependent coefficients of energy and the nonlinear equation of motion are essential points to be scrutinized by the concept of thermodynamic work, internal energy and entropy in a NIS.

The time variations of the moment of inertia, $I(t)$, corresponding to Figure 3(a) and Figure 3(b) are shown in Figure 3(c) and Figure 3(d), respectively. The corresponding changes of motion are caused by the thermodynamic work driven by up-going volatile water, resulting in time variations of $I(t)$ and $m^{*}(t)$. Since we employed piecewise continuous functions (step functions) as a mathematical model, the discontinuous changes of physical quantities at $t_{c}$ and $t_{d}$ are observed in numerical simulations. When humidity of environmental heat reservoir is saturated, DB's back-and-forth motion soon stops because of dissipation of energy, friction and viscosity, and if the simple motion around $\theta=0$ is sufficiently weak, it converges to thermodynamic equilibrium at $t \rightarrow \infty$ in a mathematical sense. In other words, if the DB's mechanical motion and the thermodynamic system cease to contribute to each other by way of heat or energy transfer, both systems will be decoupled and independently advance to mechanical and thermodynamic equilibrium states, respectively.

The concept of coupled or decoupled mechanical and thermodynamic systems is important to study mechanism of time evolutions from a NIS to thermal and mechanical equilibriums and vice versa. In case that heat or entropy flows could not produce sufficient work to affect each other, the NIS proceeds to respective equilibriums, which can be experimentally checked and may correspond to linear thermodynamics or not far from thermodynamic equilibrium. Therefore, the DB system can be in a state of thermodynamic equilibrium with the oscillation around $\theta=0$ and small differences of heat and energy as near thermodynamic equilibrium, expressed as $T \partial \mathcal{S}(\mathcal{E}, V) /\left.\partial \mathcal{E}\right|_{V, N, \ldots} \approx 1$. With the condition, the linear thermodynamics and flux equations can be discussed in the DB analysis. An example of numerical simulations of fundamental relations, (1.1)-(1.3), is specifically examined in the next section.

The fundamental macroscopic relations among the internal energy, work, heat and entropy, particle and other physical flows, are intertwined and become selfconsistent interacting quantities, and so, one should be careful to study and construct overall structural views in NISs. It becomes difficult to select macroscopic and microscopic variables, because constituent physical quantities do not maintain, for instance, $\delta \mathcal{S}=\sum_{i} \delta \mathcal{S}_{i}$. The current irreversible thermomechanical DB model exhibits "global" motion caused by changes of internal energy, entropy flow and "local" change of thermodynamic work by liquid movement in the 
glass tube and can produce the DB dynamics reasonably well. One could include the gas-phase, liquid phase and gas-liquid interface many-body interactions, local internal energies and entropies of respective phases; however, the inclusion of a local constituent variable will produce extremely complicated interactions among constituents, which makes difficult to reproduce even a DB's simple backand-forth motion.

\section{The Time-Evolution to Nonequilibrium Irreversible Thermodynamics with $(\mathcal{E}(t), T(t) \mathcal{S}(t), W(t))$}

The simple and approximate explanations to DB's motion employ pressure, temperature and volume in gas phase (Boyle-Charles' law, or the ideal gas law) to explain up-going water in a glass tube, though a DB system is not at thermodynamic equilibrium. Moreover, the concept of local equilibrium is not assumed in the thermomechanical DB model; however, the local equilibrium or linearity can be reproduced with the conditions of small entropy flow and $T \partial \mathcal{S}(\mathcal{E}, V) /\left.\partial \mathcal{E}\right|_{V, N, \cdots} \approx 1$. One should realize that the current approach is not restricted to near equilibrium. Thus, the thermomechanical model of a NIS with time-dependent thermodynamic functions, $(\mathcal{E}(t), T(t) \mathcal{S}(t), W(t))$, will help extend theoretical understandings and technical applications for nonequilibrium irreversible phenomena.

The time-dependent internal energy $\mathcal{E}(t)$ is assumed to be given by the liquid in a glass tube and the mechanical system coupled self-consistently to the time-dependent Equations (2.6) and (2.7), and thermodynamic work $W(t)$. The thermodynamic work should be a spontaneously extracted or externally measured quantity driven by heat, energy and entropy flows, restricted by environmental and mechanical states (temperature, friction, lubrication and wear, ...) and viable energy conversion technologies. The internal energy, thermodynamic work and entropy are not given by pressure, temperature and volume in gas phase, which may be interconnected with gas-liquid and liquid-phase many-body interactions. Hence, the current nonequilibrium irreversible method is not a simple alternative expression to a gas-phase method.

The entropy is discussed as the deficit function to make calculations consistent with laws of equilibrium thermodynamics [13] [14]. In the TMD, the entropy function is interpreted as the expenditure function to make calculations consistent with the total heat and energy conservation law, and we assume that the time-dependent entropy should be consistent with the first and the second laws of thermodynamics and the differential form of the time-dependent Gibbs relation in general:

$$
\mathrm{d} \mathcal{E}(t)=T(t) \mathrm{d} \mathcal{S}(t)+\mathrm{d} W(t) .
$$

The time-dependent relation between the internal energy, the thermodynamic work and the equation of motion should be consistently determined. One should note that the time-symmetry in mechanics is broken; the thermodynamic work is an externally measurable quantity, not derivable in the concept of potential 
energy. The time-evolution of total entropy, $T(t) \mathrm{d} \mathcal{S}(t)$, is the source of timedependent change, but the functional form is not necessarily to be known, which is determined to satisfy (3.1) at the end; this is the physical meaning of entropy as the expenditure function.

Reversibility at thermodynamic equilibrium means the work done by an engine must operate infinitely slowly, which is neither testable nor practical. The motion of up-going liquid is interpreted as the time-dependent thermodynamic work, considered to be driven by $T(t) \mathrm{d} \mathcal{S}(t)$, but because of indeterminable dissipation mechanisms, $T(t) \mathrm{d} \mathcal{S}(t)$ is assumed to be derived from thermodynamic work and internal energy (3.1). The time-dependent thermodynamic work, $\mathrm{d} W(t)$, is given by up-going liquid as,

$$
\mathrm{d} W(t)=\rho \sigma g z(t) \cos \theta(t) \mathrm{d} z(t),
$$

where $\rho$ is the density of liquid $\left(\mathrm{g} / \mathrm{cm}^{3}\right), \sigma$ is the area of a glass tube crosssection $\left(\mathrm{cm}^{2}\right), g=980 \mathrm{~cm} / \mathrm{s}^{2}$, and $z(t)$ is the liquid-length in a glass tube measured from the axis of rotation; $\mathrm{dz}(t)$ is the infinitesimal change, and so, the velocity of up-going liquid is given by $v_{z}(t)=\mathrm{d} z(t) / \mathrm{d} t$.

The constitutive equations of DB's irreversible motion are the coupled nonlinear equations of (2.6), (2.7), and (3.1), (3.2) to determine, $\theta(t)$ and $v_{z}(t)$. However, since the entropy flow, $T \mathrm{~d} S / \mathrm{d} t$, is given by the internal energy and work, the equation is not complete to theoretically determine the velocity of up-going liquid, $v_{z}(t)=\mathrm{d} z(t) / \mathrm{d} t$. From DB's experimental observation, one can assume the constant velocity $v_{z}(t)=v_{0}$ as an admissible solution, and the constant-velocity solution, $v_{0}$, reproduced the DB motion very well [4]. It is because one would suppose that the velocity $v_{z}(t)$ should be composed of a slightly up-and-down oscillating motion with an average constant velocity, $v_{0}$, in the form of $v_{z}(t)=v_{0}+$ small oscillations. We tested several oscillating functions with amplitudes smaller than $\pm 0.1(\mathrm{~cm})$ which should be ignored in average and satisfy the initial condition, $v_{z}(0)=v_{0}$. The test functions generated similar numerical results, indicating that the solution should be strongly stable around the solution $v_{z}(t)=v_{0}$ and supports the analysis in the paper [4]. The small oscillating velocity is compatible with changes in lines, $L_{1}, L_{2}$ and $L_{3}$ induced in the internal energy, $\mathcal{E}(\theta(t))$, shown in Figure 4, and so, $v_{z}(t)=v_{0}$ and the solution $\theta(t)$ are used for numerical simulations.

The numerical value of the internal energy corresponding to oscillations in Figure 3(a) is shown in Figure 4(a) for the 1st-drinking motion within the time-range, $10 \leq t \lesssim 60(\mathrm{~s})$. The internal energy seems a time-dependent continuous curve with discontinuous vertical lines at $t_{c}$ and $t_{d}$. However, the curves denoted as $L_{1}, L_{2}, L_{3}$ are not smooth curves; on the contrary, they are rapidly oscillating ones; for instance, $L_{3}$ in the time-range $40 \leq t \lesssim 60(\mathrm{~s})$, is shown in Figure 4(b). The rapid oscillations of the internal energy are intertwined with DB's back-and-forth oscillations and can be clearly shown when the energy flow, $\mathrm{d} \mathcal{E}(t) / \mathrm{d} t$, is calculated. With a given $v_{0}$ and a solution $\theta(t)$, one can obtain the internal energy, thermodynamic work and resultant entropy flows: $\mathrm{d} \mathcal{E}(t) / \mathrm{d} t, \mathrm{~d} W(t) / \mathrm{d} t$ and $T(t) \mathrm{d} \mathcal{S}(t) / \mathrm{d} t$, shown in Figure 5 , respectively. 


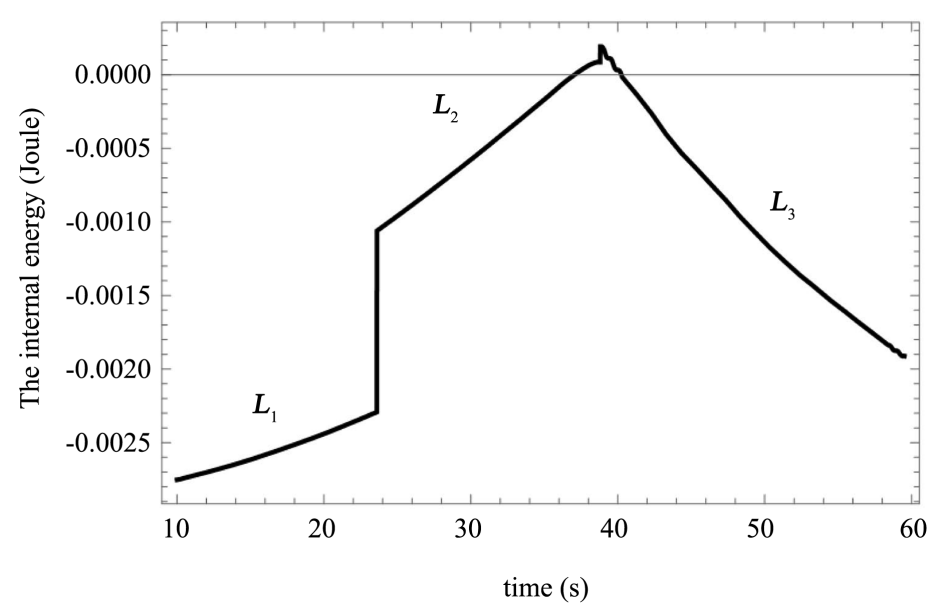

(a)

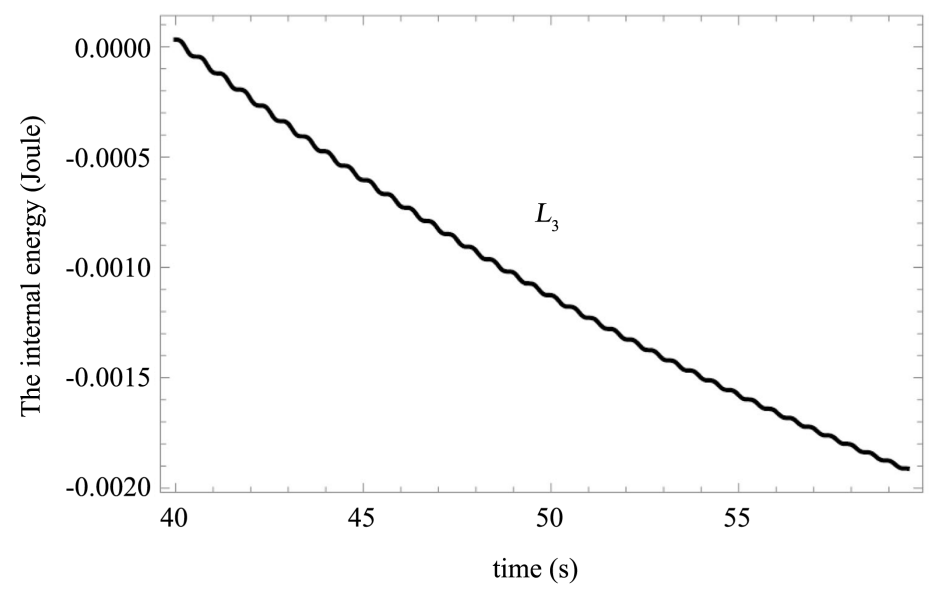

(b)

Figure 4. The time-change of internal energy for the 1st-drinking motion, Figure 3(a). Note that the lines of internal energy, $L_{1}, L_{2}, L_{3}$, are not smooth, but minute, rapidly oscillating curves. (a) The change of internal energy for the 1 st-drinking motion corresponding to Figure $3(\mathrm{a}), t_{1 c} \simeq 24.0(\mathrm{~s})$ and $t_{1 d} \simeq 38.0(\mathrm{~s})$; (b) The curves, $L_{1}, L_{2}, L_{3}$, are not smooth, but rapidly oscillating curves. A detailed, expanded observation of the line, $L_{3}$, in the time-range, $40 \leq t \leq 60$ of (a) is shown.

The thermodynamic work flow is produced in the NIS $\left(t_{c} \leq t \leq t_{d}\right)$. The energy and entropy flows, $\mathrm{d} \mathcal{E}(t) / \mathrm{d} t$ and $T(t) \mathrm{d} \mathcal{S}(t) / \mathrm{d} t$, are almost similar to each other, because the amount of power flow, $\mathrm{d} W(t) / \mathrm{d} t$, is small compared to the one of internal energy flow.

The "thermodynamic consistency" is now ready to be shown and a NIS is measured numerically. We can calculate the time-dependent thermodynamic quantity:

$$
\tau(t) \equiv \frac{T(t) \mathrm{d} \mathcal{S}(t, \mathcal{E}(t), W(t)) / \mathrm{d} t}{\mathrm{~d} \mathcal{E}(t, W(t)) / \mathrm{d} t},
$$

changing from a thermodynamic equilibrium to a NIS and vice versa; Equation (3.3) is also written as $\tau(t)=T(t)(\mathrm{d} \mathcal{S}(t) / \mathrm{d} t) /(\mathrm{d} \mathcal{E}(t) / \mathrm{d} t)$, for simplicity. 


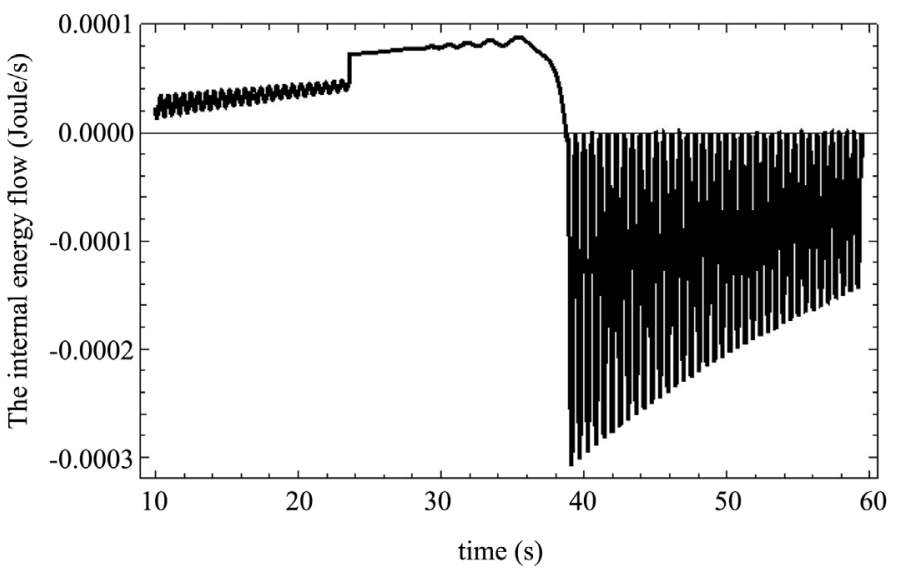

(a)

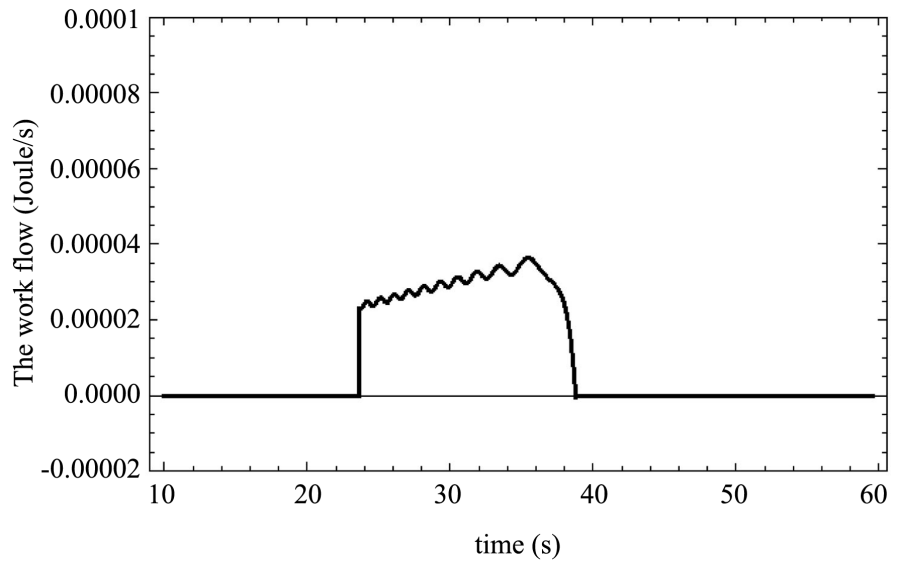

(b)

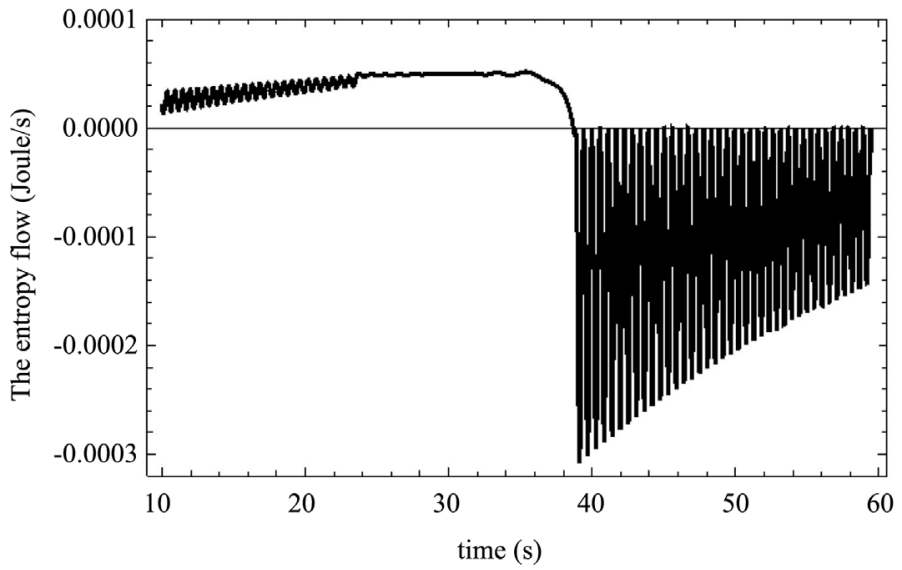

(c)

Figure 5. The internal energy, thermodynamic work and entropy flows of the 1st-drinking motion of Figure 3(a). Note that the internal energy lines in Figure 4(a) are not smooth, resulting in minute, rapidly oscillating curves. (a) The internal energy flow, $\delta \mathcal{E}(t) / \mathrm{d} t$, of the 1st-drinking motion related to Figure 3(a). $t_{1 c}=24.0(\mathrm{~s})$ and $t_{d 1}=38.8(\mathrm{~s})$; (b) The thermodynamic work flow, $\mathrm{d} W(t) / \mathrm{d} t$. The 1st-drinking motion of Figure 3(a), $\left(t_{1 c}=24.0<t<38.8=t_{d 1}(\mathrm{~s})\right)$; (c) The entropy flow, $T(t) \mathrm{d} \mathcal{S}(t) / \mathrm{d} t$, of the 1st-drinking motion derived from the total energy conservation law (3.1). 
The fundamental thermodynamic relation (1.1) is expressed by $\tau(1)=1$, and the value " 1 " is the condition of an exact thermodynamic equilibrium state, which suggests that the entropy flow is completely converted to the internal energy flow: $T(t) \mathrm{d} \mathcal{S}(t) / \mathrm{d} t=\mathrm{d} \mathcal{E}(t) / \mathrm{d} t$, and so, no time-dependent thermodynamic work exists, $\mathrm{d} W(t) / \mathrm{d} t=0$ from (3.1), which defines a thermodynamic equilibrium. If the value of $\tau(t)$ is " 0 ", it represents $T(t) \mathrm{d} \mathcal{S}(t) / \mathrm{d} t=0$, so that a thermodynamic equilibrium produces work with no transfer or dissipation of heat, resulting in the contradiction of heat and work. The consequence is compatible with the principles of R. Clausius, W. Thomson, and the other statements on thermodynamic heat and work.

Let us denote $\tilde{T}(t), \tilde{\mathcal{S}}(t), \tilde{\mathcal{E}}(t)$ for a NIS and $T, \mathcal{S}, \mathcal{E}$ for a thermodynamic equilibrium and consider the ratio of entropy flow against energy flow: $(\mathrm{d} \tilde{\mathcal{S}}(t) / \mathrm{d} t) /(\mathrm{d} \tilde{\mathcal{E}}(t) / \mathrm{d} t) \equiv \tilde{\alpha}(t)$ and $(\mathrm{d} \mathcal{S} / \mathrm{d} t) /(\mathrm{d} \mathcal{E} / \mathrm{d} t) \equiv \alpha$. Then, from (3.3), we have:

$$
\tilde{\alpha}(t)-\alpha=\frac{\tau(t)}{\tilde{T}(t)}-\frac{1}{T}=\frac{T \tau(t)-\tilde{T}(t)}{T \tilde{T}(t)} .
$$

It shows that $\tilde{\alpha}(t)-\alpha>0$, or equivalently, $\tilde{\alpha}(t) / \alpha>1$ results in $\tilde{T}(t)<T \tau(t)$. We interpret it as the amount of heat-entropy "flow-out", compared to the thermodynamic equilibrium quantity $\alpha$. Since the heat-entropy flows out of the system faster than the internal energy flow $\mathrm{d} \tilde{\mathcal{E}}(t) / \mathrm{d} t$, the temperature $\tilde{T}(t)$ in the NIS becomes lower than $T \tau(t)$. Similarly, $\tilde{\alpha}(t)-\alpha<0$ or equivalently, $\tilde{\alpha}(t) / \alpha<1$ (the amount of heat-entropy "flow-in") results in $\tilde{T}(t)>T \tau(t)$. In this case, because the heat-entropy flows in the system, the temperature $\tilde{T}(t)$ in the NIS becomes higher than $T \tau(t)$.

When $\tilde{T}(t)$ is lower than $T$, the nonequilibrium temperature $\tilde{T}(t)$ will gradually increase and progress to $T,(\tilde{T}(t) \nearrow T)$, with $\tilde{T}(t) / T<\tau(t) \rightarrow 1$, and similarly, when $\tilde{T}(t)$ is higher than $T$, the temperature will gradually decrease and progress to $T(\tilde{T}(t) \searrow T)$ with $\tilde{T}(t) / T>\tau(t) \rightarrow 1$. The measure $\tau(t)$ for a NIS is consistent with observed experimental changes of $\tilde{T}(t)$ and $T$. The time-dependent temperature, $\tau(t)$, is constructed by the ratio of entropy transfer against the energy transfer, and it explains one of the reasons why the concept of temperature is fundamental in physics, as well as engineering and sciences in general. We will define $\tau(t)$ as the nonequilibrium temperature, or a measure of a NIS, which is one of the fundamental results of the thermomechanical dynamics (TMD).

Now, the second law of thermodynamics, the concept of entropy, can be explained by the statement: the nonequilibrium temperature $\tau(t)$ is a positive definite quantity: $\tau(t)>0$. This is explained such that if a system is not in an equilibrium state, the entropy-flow and the energy-flow will emerge and progress to an equilibrium state. The nonequilibrium temperature converges to an equilibrium temperature $(\tau(t) \rightarrow 1): \tilde{T}(t) \rightarrow T$, which will distinguish two cases: $\tau(t)<1$ means that $\tilde{T}(t)$ is lower than $T$, and $\tau(t)>1$ means that $\tilde{T}(t)$ is higher than $T$. The result is physically transparent meaning that if the tempera- 
ture $\tilde{T}(t)$ in a local nonequilibrium system is initially lower than the equilibrium temperature $T$, the entropy will increase to the maximum entropy at equilibrium, but if the temperature $\tilde{T}(t)$ in a local nonequilibrium system is initially higher than the equilibrium temperature $T$, the entropy will decrease to the minimum entropy at equilibrium. One should note that the revolution to extrema of entropy depends on the difference between $\tilde{T}(t)$ of a NIS and the corresponding equilibrium temperature $T$.

The internal energy, $\mathcal{E}(t)$, and the energy-flow, $\mathrm{d} \mathcal{E}(t) / \mathrm{d} t$, of the 1 st and the 2nd dipping motions are shown in Figure 6(a) and Figure 6(b), and the entropy-flow, $T(t) \mathrm{d} \mathcal{S}(t) / \mathrm{d} t$, is similar to the internal energy given by Figure $6(\mathrm{~b})$. The current thermomechanical calculation of $\tau(t)$ in the DB system results in $\tau(t)<1$, shown in Figure 7 , which concludes that the temperature inside the DB is lower than that of external equilibrium environment as one can readily check from the DB experiment independently. The numerical results of $\tau(t)$ for the 1st and the 2nd dipping motions are shown in Figure 7(a) and Figure 7(b), respectively.

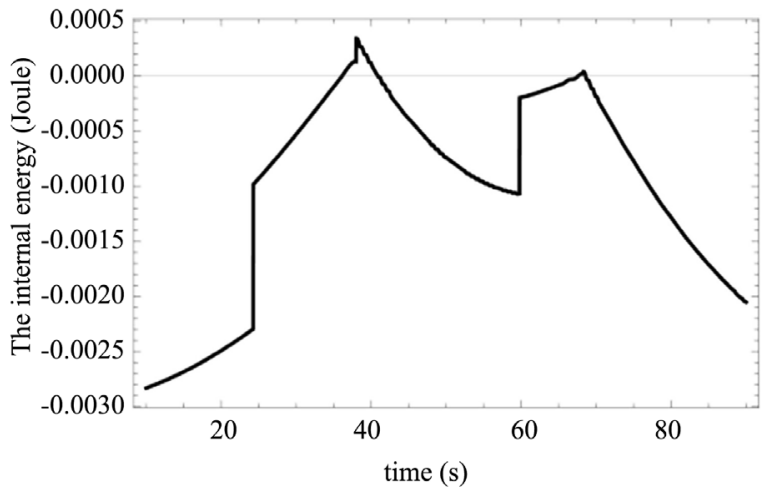

(a)

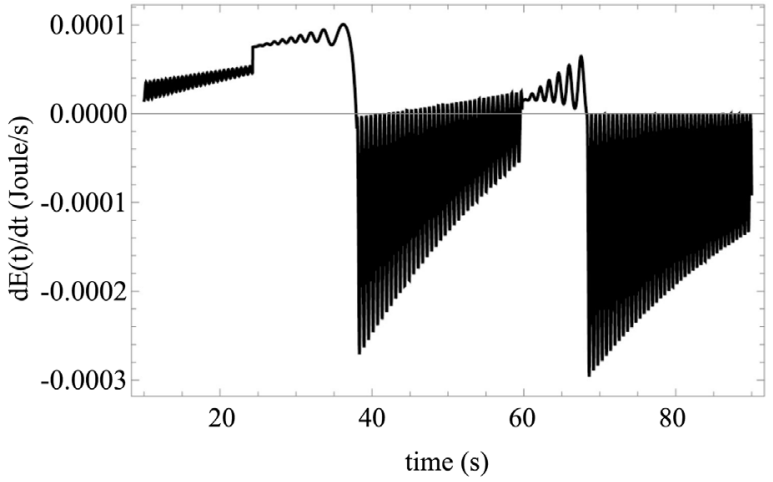

(b)

Figure 6. The time-dependent internal energy, $\mathcal{E}(t)$, of the 1 st and the 2 nd drinking motions and the internal energy flow, $\delta \mathcal{E}(t) / \mathrm{d} t$. (a) The time-dependent internal energy, $\mathcal{E}(t)$, for the 1 st and 2 nd drinking motions of Figure $3(\mathrm{~b}) . t_{1 c} \simeq 24.0(\mathrm{~s})$ and $t_{1 d} \simeq 38.0(\mathrm{~s}) ; t_{2 c} \simeq 60.5(\mathrm{~s})$ and $t_{2 d} \simeq 68.3(\mathrm{~s}) ;(\mathrm{b})$ The internal energy flow, $\delta \mathcal{E}(t) / \mathrm{d} t$, for the 1 st and the 2nd drinking motions of Figure $3(\mathrm{~b})$. 


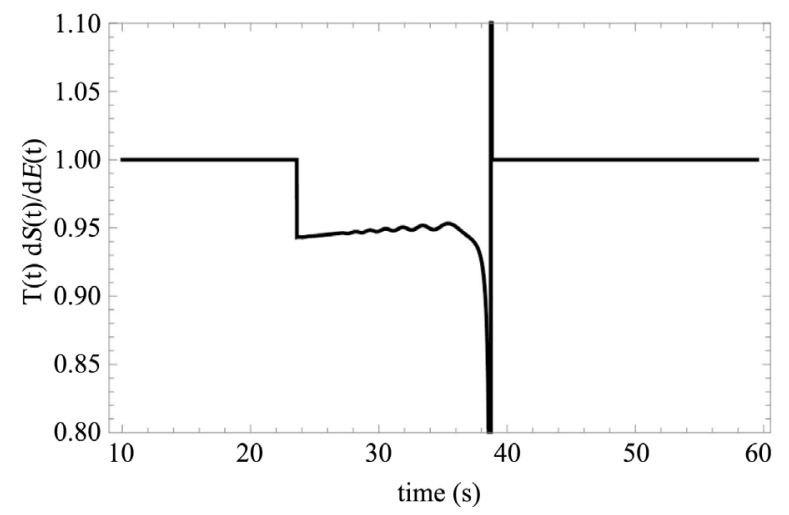

(a)

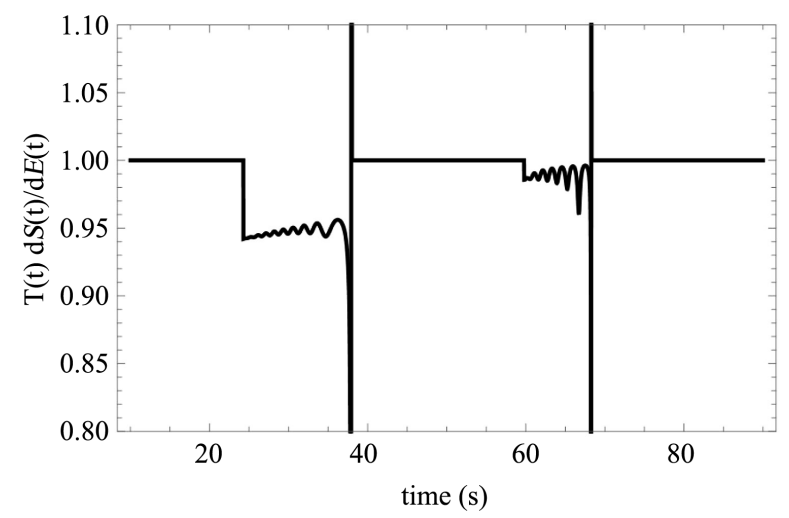

(b)

Figure 7. The time-evolution of $T(t)(\mathrm{d} \mathcal{S}(t) / \mathrm{d} t) /(\mathrm{d} \mathcal{E}(t) / \mathrm{d} t)$, and thermodynamic consistency. (a) The time-evolution of the entropy and internal energy flows,

$T(t)(\mathrm{d} \mathcal{S}(t) / \mathrm{d} t) /(\mathrm{d} \mathcal{E}(t) / \mathrm{d} t)$, for the drinking motion of Figure $3(\mathrm{~b}) . t_{1 c} \simeq 24.0(\mathrm{~s})$ and $t_{d 1} \simeq 38.8(\mathrm{~s})$; (b) The time-evolution of the entropy and internal energy flows,

$T(t)(\mathrm{d} \mathcal{S}(t) / \mathrm{d} t) /(\mathrm{d} \mathcal{E}(t) / \mathrm{d} t)$, for the drinking motion of Figure $3(\mathrm{~b}) . t_{1 c} \simeq 24.0(\mathrm{~s})$ and $t_{1 d} \simeq 38.0(\mathrm{~s}) ; t_{2 c} \simeq 60.5(\mathrm{~s})$ and $t_{2 d} \simeq 68.3(\mathrm{~s})$.

The DB's state is in mechanical and thermodynamic equilibriums at the outset and then, the transition to a NIS arises at $t_{c} \simeq 24.0(\mathrm{~s})$. The straight horizontal line represents a thermodynamic equilibrium, and the behaviors at $t_{c}$ and $t_{d}$ are only discontinuous in our model simulations because of the use of piecewise continuous step functions for the simplification of the physical mechanism at $t_{c}$ and $t_{d}$; for instance, one can integrate the heat-conduction mechanism at $t_{c}$ and $t_{d}$, in order to elaborate the conduction of time-dependent temperature. The nonequilibrium temperature, $\tau(t)$, changes rather like a heat-wave pulse function, and one should note that $\tau(t)$ is not necessary a monotonically changing function, except at a near-equilibrium. After the DB's 1st-drinking at $t_{1 d} \simeq 38.8(\mathrm{~s})$, the state decouples to equilibrium states and returns back to oscillations around $\theta=0$ and $\operatorname{T} \partial \mathcal{S}(\mathcal{E}, V) /\left.\partial \mathcal{E}\right|_{V, N, \cdots}=1,\left(t_{1 d}<t\right)$. The second NIS arises in the time period, $60.5<t<68.3(\mathrm{~s})$, and the nonequilibrium temperature, $\tau(t)$, shows oscillating evolutions to an equilibrium temperature, as shown in Figure 7 (b). 
The thermal conduction mechanism could be integrated in the current thermomechanical method. There would be so many applications, for example, the irreversible processes such as friction, ignition, combustion and detonation mechanisms [30] [31] [32] [33] [34], mechanical heat engines, quantum heat engines [35] [36] [37] [38], solar-powered, high and low temperature differential Stirling engines [39] [40] [41], quantum thermodynamic systems [42] [43] [44]. The mechanism of drinking bird is more sophisticated and fundamental than that of Stirling engines, because it spontaneously converts thermal energy into mechanical energy and thermodynamic work by way of the concept of entropy-flow. Technological applications and theoretical consistency should be investigated further when one constructs thermo-quantum mechanics (TQM), thermo-electrodynamics (TED) and so forth. The results of TMD will be helpful to construct consistent thermal dynamics.

\section{The Thermomechanical Dynamics (TMD) for the Nonequilibrium Irreversible Thermodynamics}

The method of thermomechanical dynamics by employing a drinking bird mechanism has shown useful concepts and specific results for nonequilibrium irreversible states, which is different from probability theory and distribution function method in kinetic theories. The results and the foundations of TMD are summarized in this section.

The self-consistent analysis of the DB system would simultaneously direct one to confront emergent phenomena: the coupling or decoupling between mechanics and thermodynamics. It is never a simple, complementary mechanism explained by mechanics and thermodynamics. It demands the transition from a time-symmetric state to a time-symmetry-broken state and vice versa. Both mechanics and thermodynamics will break down, and a new state emerges as a NIS. The time-symmetry of Hamiltonian is broken, resulting in non-conservation of energy, but instead, the total heat and energy is conserved in the time range, $t_{c}<t<t_{d}$. Mathematically speaking, the equation of motion for DB's mechanical equilibrium is given by a nonlinear differential equation with constant coefficients, but the equation of motion in the corresponding NIS is given by the same nonlinear differential equation with time-dependent coefficients. The time-dependent nonlinear equation has independent solutions not derivable from the one with constant coefficients. The transformation to a nonlinear differential equation with time-dependent coefficients might correspond to a mathematical way of expressing a transition from a physical phase to a new emergent phenomenon. The nonlinear differential equations with constant and time-dependent coefficients must be mathematically categorized in a different class of nonlinear equations. This is an important mathematical property discovered in the analysis of the DB system.

The concept of the local equilibrium and the near-equilibrium state, the local microscopic reversibility, the linearity of fluxes and forces in transport processes [45] [46] [47] [48] are not necessarily claimed at the outset. The conditions of 
linearity and near equilibrium can be extracted from $\mathrm{d} \mathcal{E}(t) / \mathrm{d} t, \mathrm{~d} W(t) / \mathrm{d} t$, and $T(t) \mathrm{d} \mathcal{S}(t) / \mathrm{d} t$, with the condition $T \partial \mathcal{S}(\mathcal{E}, V) /\left.\partial \mathcal{E}\right|_{V, \ldots} \simeq 1$ in the DB system. The ratio of the internal energy and the heat-entropy flows, (14), will define a nonequilibrium temperature and a measure of how far the system has deviated from a corresponding thermodynamic equilibrium.

Based on discussions and results so far, the methodology and the fundamental postulates of TMD for heat engines and transport mechanism will be summarized as follows:

1) If thermal and mechanical equilibriums coexist like a system of heat engine, time-dependent Hamiltonian (or Lagrangian) and equations of dissipative motion (with friction and friction-induced time-dependent phenomena) must be constructed, which should be identified as the time-dependent internal energy and the corresponding nonlinear equation of dissipating motion.

2) The work, $d W(t)$, must be an externally measurable quantity and defined against the dissipative mechanical motion proceeding to mechanical equilibrium, given by the inner product: $F(t) \cdot \mathrm{dl}(t)(\mathrm{d} l(t)$; the work-direction increment), or, by available energy transformation technologies such as heat-electricity, charge-energy transfer and so forth.

a) The time-dependent force $F(t)$ is local, not given by a mechanical potential defined in a given Hamiltonian. The time-symmetry of mechanical Hamiltonian is broken by the generation of time-dependent work, $\mathrm{d} W(t)$.

b) The time-dependent Hamiltonian $H(t)$ identified as the internal energy $\mathcal{E}(t)$, and the time-dependent thermodynamic work $\mathrm{d} W(t)$, must maintain the total heat-energy conservation law: $\mathrm{d} \mathcal{E}(t)=T(t) \mathrm{d} \mathcal{S}(t)+\mathrm{d} W(t)$, and the differential form of the time-dependent Gibbs relation in general. The total entropy $T(t) \mathrm{d} \mathcal{S}(t)$ is interpreted as the expenditure function to make TMD consistent with the total heat-energy conservation law.

3) The measure of a NIS, or the nonequilibrium temperature is defined by:

$$
\tau(t)=\frac{T(t) \mathrm{d} \mathcal{S}(t) / \mathrm{d} t}{\mathrm{~d} \mathcal{E}(t) / \mathrm{d} t} .
$$

a) Thermodynamic equilibrium, the relation between internal energy and work when $\mathrm{d} S(t) / \mathrm{d} t=0$, and the principle of entropy correspond to $\tau(t)=1$, $\tau(t)=0$, and $\tau(t)>0$, respectively.

b) The thermal and mechanical equilibriums can exist when the entropy flow could not affect the internal energy to produce observable work. Near equilibriums and not-far-from equilibriums are studied by

$\tau(t)=T(t)(\mathrm{d} \mathcal{S} / \mathrm{d} t) /(\mathrm{d} \mathcal{E} / \mathrm{d} t) \sim 1$.

The postulate discussed above is applicable to examine heat engines and NISs, and it should be extended by including heat-conduction, ignition and detonation, friction and diffusion mechanism for elaboration and applied to self-lubricating, self-organizing smart materials and so forth [30] [31] [32] [33]. The application of "thermodynamic consistency" to mechanism of the heat-conduction would be very helpful, and the fields of research with TMD would improve the analysis of 
physics of heat-conduction mechanism, heat-source and temperature, which may enhance profound revolutions in vast fields of science. The analysis of thermodynamic consistency should be applied to systems of electrodynamics, quantum mechanics, quantum electrodynamics (QED), nuclear quantum-hadrodynamics (QHD) [49]-[55]. The concept of heat engine should be expanded and applied in general from macroscopic to microscopic quantum energy scales, such as solar, wind, hydroelectric, thermal, nuclear energy, and so forth.

\section{Conclusions and Perspectives of Scientific Ways of Thinking}

We have shown the theory of nonequilibrium irreversible thermodynamics and the mechanism of a drinking bird as a rigorous and fascinating device and obtained new mathematical and physical results: the coupling and decoupling of thermal and mechanical states, symmetry-breaking and restoration, bifurcation solutions and independent solutions of nonlinear equations with time-dependent coefficients, the measure of nonequilibrium states and the postulate of thermomechanical dynamics of nonequilibrium irreversible phenomena.

A DB toy is composed of a many-body system of interactions among liquid, gas and interface of gas-liquid. The TMD demands that thermomechanical variables should be carefully and optimally chosen to be minimal because constituent variables will be self-consistently intertwined with others. Hence, one should be careful that the partition to many constituents and the direct sum of constituents usually discussed at thermodynamic equilibrium are not allowed in NISs. The measure of irreversibility demands that the time-dependent fluctuation of entropy should be evaluated relatively to the time-dependent internal energy. It could be physically reasonable that the measurement-induced fluctuations, ignition and detonation, friction and diffusion mechanism, extremely high-temperature gradients at small scales and short periods of time should be measured and studied by the ratio of entropy and internal energy flows. Heat conduction and thermal management in modern nanostructure technologies at molecular and atomic levels may require new kinds of experiments and thermomechanical methods for measurement and analysis [56].

In the paper [4], we discussed the fundamental property and logical structure that scientific theories and models must maintain and summarized as the triad condition for models and theories. reproducibility, testability and self-consistency. The relative word like "beauty and simplicity" is neither sufficient nor useful, because it depends on one's taste and often leads to a partial idolization, which is against the scientific truth. The scientific spirits and minds we can trust are in endeavors to maintain and achieve the triad condition in scientific activities. The current paper is on the self-consistency of the DB system as a transport engine, whose motion is essentially in a NIS. Hence, the analysis of self-consistency of the DB system directly interconnects the current theoretical method to fundamental laws of thermodynamics. With the current paper and the papers [4] [5], we have specifically shown an example that the DB mechanism maintains the 
triad condition. This is the beginning of extensions to more general transport phenomena, and as long as physics and sciences, in general, can maintain the concept of the triad condition, they could be open authorities, useful for human society and nature.

The mechanism and fundamental characteristics of a drinking bird are the scientific motivation for our writing a series of papers, but in addition, it directed us to scientific ways of thinking, supported and encouraged us while studying a physical model of heat engines. The scientific views that should be fundamental to discuss here are: reductionism, structuralism and emergentism (or emergent dynamics). The intuitive understandings of the three views are essential for scientists as a whole. The successful reductionist views but associated contradictions and conceptual problems induced in diverse fields of science, nature and human society, are well summarized in the papers [57] [58] [59]. The reduction hypothesis is in the existence of basic ultimate building blocks, and the whole structure reduces to a complete summation of constituent building blocks or particles, but the hypothesis is not even true in the nonequilibrium irreversible mechanism of a drinking bird. The central claim of structuralism is that the wholeness is neither reducible, explainable, nor predictable as the sum of lower-level constituents. The structuralist views appeared against reductionist ones, especially in the fields of physics, micro- and macro-biology, sociology, anthropology and linguistics [59] [60] [61] [62].

The wholeness is an abstract concept difficult to define and often seriously misunderstood in sociology and politics. For simplicity, let us consider the equation of motion and Hamiltonian of a simple pendulum shown in (2.4) and assume that the basic building blocks for the pendulum Hamiltonian are length and mass (or moment of inertia), gravity and angle, $(l, m, g, \theta)$. The wholeness appears as symmetries and the energy conservation law for basic building blocks. Then, one would introduce the coupling of many pendulums with possible potential interactions to explain dynamics of the system. However, it is generally known that many-body interaction phenomena such as superconductivity, collective phenomena, thermodynamic consistency [63] [64] [65] [66], and frictions, shear and wear, fluctuations of heat and entropy, phase transitions and so forth, are not simply explainable from constants and constituents of corresponding Hamiltonians. If constants of a pendulum, $(l, m, g)$, are assumed to change with time, they would have unexpected effects on solutions as a whole, but it is not known and difficult to know that what values and combinations of constants, when and how critical many-body phase-transition phenomena will emerge. This kind of fundamental problem is similarly discovered in DB's mechanism as the emergence of bifurcation solutions and independent continuous solutions in nonlinear differential equations with time-dependent coefficients, related to the transition between thermodynamic equilibrium and a nonequilibrium irreversible state.

As the reductionist and structuralist views are considered to be synthesized in physics as the rigorous methodology of Lagrangian and Hamiltonian mechanics, 
the view against reductionism in physics has gradually surfaced from microscopic many-body interactions [67] [68] to intermolecular, macroscopic interactions in order to explain emergent phenomena [57] [58] [59]. The emergentism is also well documented so that it suffices to quote some of them. "An emergent property or phenomenon is usually defined as one that arises out of lower-level constituents but is neither reducible, explainable, nor predictable from them" [69] [70], and "The ability to reduce everything to simple fundamental laws does not imply the ability to start from those laws and reconstruct the universe... The constructionist hypothesis breaks down when confronted with the twin difficulties of scale and complexity... we can see the whole becomes not only more than but very different from the sum of its parts" [59].

The examples against reductionist views are abundant in condensed matter physics, such as superconductivity, phase-transition phenomena, symmetrybreaking, effective field theories in hadron dynamics and so forth. The assertion coincides with one of the DB's claims for nonequilibrium irreversible states: $d \mathcal{S} \neq \sum_{i} d \mathcal{S}_{i}$, with the indication that the relation, $d \mathcal{S}=\sum_{i} d \mathcal{S}_{i}$, is only true at thermodynamic equilibrium and a quantum statistical ensemble when interactions are negligible among constituents. The logical character supports the current method of TMD for the nonequilibrium irreversible dynamics. The reductionist way of thinking may conclude that even emergent phenomena and structuralism will be resolved from a theory of everything, which comes from typical misunderstandings of emergent phenomena and structuralism. Although the reductionist method has contributed to science and technologies, "The existence of emergent phenomena undermines the kind of reductionism that is presupposed in the search for a theory of everything" [69] [70].

The technologies and scientific ways of thinking have contributed to human societies and understandings of nature from microscopic to macroscopic phenomena, liberating our mind and spirit from oppressions and limitations. The achievement of sciences and technologies is powerful and gigantic, but a big science with sociology, politics and group-oriented authoritative ways of thinking may suppress our scientific mind and spirit [71]. The big science and mainstream ideas could be good or bad like a double-edged sword. Hence, scientists must be extremely careful about the logic and truth of science in the future, against politics and group-oriented authorities. The scientific spirit and ideas should be rigid and strict, but simultaneously, open and flexible. In addition to the triad condition for models and theories, the triad condition for scientific approaches: reductionism, structuralism and emergentism, will be useful to check scientific ways of thinking, which is essential knowledge for science and logical mind of all people.

\section{Conflicts of Interest}

The authors declare no conflicts of interest regarding the publication of this paper. 


\section{References}

[1] Bagnoli, F., Guarino, A. and Pacini, G. (2019) Teaching Physics by Magic. Physics Education, 54, Article No. 015025. https://doi.org/10.1088/1361-6552/aaed62

[2] Güémez, J. and Fiolhais, M. (2013) From Mechanics to Thermodynamics-Analysis of Selected Examples. European Journal of Physics, 34, 345-357. https://doi.org/10.1088/0143-0807/34/2/345

[3] Bucky, P.A. (1992) The Private Albert Einstein. Andrews and McMeel, Kansas City.

[4] Uechi, S.T., Uechi, H. and Nishimura, A. (2019) The Analysis of Thermomechanical Periodic Motions of a Drinking Bird. World Journal of Engineering and Technology, 7, 559-571. https://doi.org/10.4236/wjet.2019.74040

[5] Uechi, H. and Uechi, S.T. (2020) Thermoelectric Energy Conversion of a Drinking Bird by Disk-Magnet Electromagnetic Induction. World Journal of Engineering and Technology, 8, 204-216. https://doi.org/10.4236/wjet.2020.82017

[6] Zhang, D., Zheng, X. and Ventra, M.D. (2019) Local Temperatures Out of Equilibrium. Physics Reports, 830, 1-66. https://doi.org/10.1016/j.physrep.2019.10.003

[7] Lieb, E.H. and Yngvason, J. (1999) The Physics and Mathematics of the Second Law of Thermodynamics. Physics Reports, 310, 1-96. https://doi.org/10.1016/S0370-1573(98)00082-9

[8] Førland, K.S., Førland, T. and Ratkje, S.K. (1988) Irreversible Thermodynamics: Theory and Applications. John Wiley \& Sons, Hoboken.

[9] Guggenheim, E.A. (1967) Thermodynamics. Elsevier Science Pub., North-Holland.

[10] Demirel, Y. (2014) Nonequilibrium Thermodynamics. Elsevier, Amsterdam.

[11] Yourgrau, W., Merwe, A.V.D. and Raw, G. (1982) Treatize on Irreversible and Statistical Thermophysics. Dover Publications, New York.

[12] Kosloff, R. (2013) Quantum Thermodynamics: A Dynamical Viewpoint. Entropy, 15, 2100-2128. https://doi.org/10.3390/e15062100

[13] Mahler, G. (2015) Quantum Thermodynamic Processes. CRC Press, Boca Raton.

[14] Honig, J.M. (2007) Thermodynamics: Principles Characterizing Physical and Chemical Processes. Elsevier Science \& Technology Books, Tokyo.

[15] Dewar, R.C., Lineweaver, C.H., Niven, R.K. and Regenauer-Lieb, K. (2014) Beyond the Second Law. Springer, New York. https://doi.org/10.1007/978-3-642-40154-1

[16] Munõz, A.G. (2013) Photoelectrochemical Solar Conversion Systems. CRC Press Taylor \& Francis Group, New York.

[17] Brinkert, K. (2018) Energy Conversion in Natural and Artificial Photosynthesis. Springer, New York. https://doi.org/10.1007/978-3-319-77980-5

[18] Santillán, M. (2014) Chemical Kinetics, Stochastic Processes, and Irreversible Thermodynamics. Springer, New York. https://doi.org/10.1007/978-3-319-06689-9

[19] Rubin, A. and Riznichenko, G. (2014) Mathematical Biophysics. Springer, New York. https://doi.org/10.1007/978-1-4614-8702-9

[20] Stefanucci, G. and Leeuwen, R.V. (2013) Nonequilibrium Many-Body Theory of Quantum Systems. Cambridge University Press, Cambridge.

[21] Lu, K. (2014) Materials in Energy Conversion, Harvesting, and Storage. John Wiley \& Sons, Hoboken. https://doi.org/10.1002/9781118892374

[22] Karady, G.G. and Holbert, K.E. (2013) Electrical Energy Conversion and Transport. IEEE, John Wiley, Hoboken. https://doi.org/10.1002/9781118498057

[23] Pleskov, Y.U. and Gurevich, Y.Y. (1986) Semiconductor Photoelectrochemistry. 
Consultant Bureau, New York. https://doi.org/10.1007/978-1-4684-9078-7

[24] Uechi, S.T. and Uechi, H. (2018) The Profiling of International Roughness Index (IRI) Based on Lagrangian Method. World Journal of Engineering and Technology, 6, 885-902. https://doi.org/10.4236/wjet.2018.64059

[25] Uechi, S.T. and Uechi, H. (2019) A Mechanical Vibration-Induced Electric Energy Generation (MVEG) and Applications to Ride Quality of Vehicles and International Roughness Index (IRI). Studies in Engineering and Technology, 6, 59-69. https://doi.org/10.11114/set.v6i1.4301

[26] Wells, D.A. (1967) Lagrangian Dynamics. McGraw-Hill, Boston.

[27] Goldstein, H. (1980) Classical Mechanics. Addison-Wesley, Tokyo.

[28] Strogatz, S.H. (1994) Nonlinear Dynamics and Chaos: With Applications to Physics, Biology, Chemistry, and Engineering. Westview Press, Boulder Colorado.

[29] Smith, H.L. and Waltman, P. (1995) The Theory of the Chemostat. Cambridge University Press, Cambridge. https://doi.org/10.1017/CBO9780511530043

[30] Cole, K.D., Beck, J.V., Haji-Sheikh, A. and Litkouhi, B. (2011) Heat Conduction Using Green's Functions. Taylor and Francis Group, LLC, New York.

https://doi.org/10.1201/9781439895214

[31] Rubtsov, N.M., Seplyarskii, B.S. and Alymov, M.I. (2017) Ignition and Wave Processes in Combustion of Solids. Springer, New York. https://doi.org/10.1007/978-3-319-56508-8

[32] Nosonovsky, M. and Mortazavi, V. (2014) Friction-Induced Vibrations and SelfOrganization. Taylor \& Francis Group, Boca Raton. https://doi.org/10.1201/b15470

[33] Terao, K. (2007) Irreversible Phenomena. Springer, New York.

[34] Jou, D., Casas-Vazquez, J. and Lebon, G. (2010) Extended Irreversible Thermodynamics. Springer, New York.

[35] Alipour, S., Benatti, F., Bakhshinezhad, F., Afsary, M., Marcantoni, S. and Rezakhani, A.T. (2016) Correlations in Quantum Thermodynamics: Heat, Work, and Entropy Production. Scientific Reports, 6, Article No. 35568. https://doi.org/10.1038/srep35568

[36] Weimer, H., Henrich, M.J., Rempp, F., Schröder, H. and Mahler, G. (2008) Local Effective Dynamics of Quantum Systems: A Generalized Approach to Work and Heat. EPL, 83, 30008. https://doi.org/10.1209/0295-5075/83/30008

[37] Kieu, T.D. (2004) The Second Law, Maxwell's Demon, and Work Derivable from Quantum Heat Engines. Physical Review Letters, 93, 140403.

https://doi.org/10.1103/PhysRevLett.93.140403

[38] Denur, J. (2019) Improving Heat-Engine Performance via High-Temperature Recharge. InTechOpen, London. https://doi.org/10.5772/intechopen.89913

[39] Senft, J.R. (1993) An Introduction to Stirling Engines. Moriya Press, River Falls.

[40] Senft, J.R. (1996) An Introduction to Low Temperature Differential Stirling Engines. Moriya Press, River Falls.

[41] Izumida, Y. (2020) Quasilinear Irreversible Thermodynamics of a Low-Temperature-Differential Kinematic Stirling Heat Engine. Physical Review E, 102, 012142. https://doi.org/10.1103/PhysRevE.102.012142

[42] Klatzow, J., Becker, J.N., Ledingham, P.M., et al. (2019) Experimental Demonstration of Quantum Effects in the Operation of Microscopic Heat Engines. Physical Review Letters, 122, 110601. https://doi.org/10.1103/PhysRevLett.122.110601

[43] Kongtragool, B. and Wongwises, S. (2003) Review of Solar-Powered Stirling En- 
gines and Low Temperature Differential Stirling Engines. Renewable and Sustainable Energy Reviews, 7, 131-154. https://doi.org/10.1016/S1364-0321(02)00053-9

[44] Dou, W., Bätge, J., Levy, A. and Thoss, M. (2020) Universal Approach to Quantum Thermodynamics of Strongly Coupled Systems under Nonequilibrium Conditions and External Driving. Physical Review B, 101, 184304.

https://doi.org/10.1103/PhysRevB.101.184304

[45] Jiménez de Cisneros, B. and Hernández, A.C. (2008) Coupled Heat Devices in Linear Irreversible Thermodynamics. Physical Review E, 77, 041127.

https://doi.org/10.1103/PhysRevE.77.041127

[46] Izumida, Y. and Okuda, K. (2009) Onsager Coefficients of a Finite-Time Carnot Cycle. Physical Review E, 80, 021121. https://doi.org/10.1103/PhysRevE.80.021121

[47] Izumida, Y., Okuda, K., Roco, J.M.M. and Hernández, A.C. (2015) Heat Devices in Nonlinear Irreversible Thermodynamics. Physical Review E, 91, 052140.

https://doi.org/10.1103/PhysRevE.91.052140

[48] Heimburg, T. (2017) Linear Nonequilibrium Thermodynamics of Reversible Periodic Processes and Chemical Oscillations. Physical Chemistry Chemical Physics, 19, 17331-17341. https://doi.org/10.1039/C7CP02189E

[49] Serot, B.D. and Walecka, J.D. (1986) Advances in Nuclear Physics (Edited by Negele, J.W. and Vogt, E.). Plenum, New York, Vol. 16.

[50] Serot, B.D. (1992) Quantum Hadrodynamics. Reports on Progress in Physics, 55, 1855. https://doi.org/10.1088/0034-4885/55/11/001

[51] Furnstahl, R.J. and Serot, B.D. (1990) Covariant Mean-Field Calculations of FiniteTemperature Nuclear Matter. Physical Review C, 41, 262.

https://doi.org/10.1103/PhysRevC.41.262

[52] Furnstahl, R.J. and Serot, B.D. (1991) Covariant Feynman Rules at Finite Temperature: Time-Path Formulation. Physical Review C, 44, 2141-2174.

https://doi.org/10.1103/PhysRevC.44.2141

[53] Uechi, H. (2004) The Theory of Conserving Approximations and the Density Functional Theory in Approximations for Nuclear Matter. Progress of Theoretical Physics, 111, 525-543. https://doi.org/10.1143/PTP.111.525

[54] Uechi, H. (2018) The Chiral Dirac-Hartree-Fock Approximation in QHD with Scalar Vertex Corrections. Open Access Library Journal, 5, e4739.

https://doi.org/10.4236/oalib.1104739

[55] Uechi, S.T. and Uechi, H. (2016) Landau Theory of Fermi Liquid in a Relativistic Nonlinear $(\sigma, \omega)$ Model at Finite Temperature. Open Access Library Journal, 3, Article No. e2757.

[56] Zhang, Z.M. (2020) Nano/Microscale Heat Transfer. Springer, New York. https://doi.org/10.1007/978-3-030-45039-7

[57] Bhom, D. (2002) Wholeness and the Implicate Order. Routledge Classics, London and New York.

[58] Schweber, S.S. (1993) Physics, Community and the Crisis in Physical Theory. Physics Today, 46, 34. https://doi.org/10.1063/1.881368

[59] Anderson, P.W. (1972) More Is Different, Broken Symmetry and the Nature of Hierarchical Structure of Science. Science, New Series, 177, 393-396.

https://doi.org/10.1126/science.177.4047.393

[60] Uechi, H., Uechi, S.T. and Serot, B.D. (2012) Chaps. 1-3 and 5-6, in Neutron Stars: The Aspect of High Density Matter, Equations of State and Observables. Nova Science Publishers, New York. 
[61] Uechi, S.T. and Uechi, H. (2015) Density-Dependent Properties of Hadronic Matter in an Extended Chiral $(\sigma, \pi, \omega)$ Mean-Field Model. Open Access Library Journal, 2, Article No. e2011. https://doi.org/10.4236/oalib.1102011

[62] Piajet, J. (1970) Structuralism. Basic Books, New York.

[63] Fetter, A.L. and Walecka, J.D. (2003) Quantum Theory of Many-Particle Systems. Dover Pub., New York.

[64] Zagoskin, A.M. (1998) Quantum Theory of Many-Body Systems. Springer, New York. https://doi.org/10.1007/978-1-4612-0595-1

[65] Mattuck, R.D. (1976) A Guide to Feynmann Diagrams in the Many-Body Problem. McGraw-Hill, Boston.

[66] Uechi, S.T. and Uechi, H. (2017) Self-Consistent Many-Body Theory and Nuclear Matter in a Chiral Dirac-Hartree-Fock Approximation. Quarterly Physics Review, 3, No. 3 .

[67] Israelachivili, J.N. (1992) Intermolecular and Surface Forces. Academic Press, Cambridge.

[68] Pollock, D.D. (1991) Thermocouples, Theory and Properties. CRC Press, Boca Raton.

[69] Morrison, M. (2012) Emergent Physics and Micro-Ontology. Philosophy of Science, 79, 141-166. https://doi.org/10.1086/663240

[70] Morrison, M. (2006) Emergence, Reduction, and Theoretical Principles: Rethinking Fundamentalism. Philosophy of Science, 73, No. 5. https://doi.org/10.1086/518746

[71] Smolin, L. (2006) The Troubles with Physics. Spin Networks, New York. 